\title{
The Association of Hypoglycemia
} Assessed by Continuous Glucose Monitoring With Cardiovascular Outcomes and Mortality in Patients With Type 2 Diabetes

OPEN ACCESS

Edited by:

Jie Chen,

Xiamen University, China

Reviewed by:

Youlian Wang

Jiangxi Provincial People's

Hospital, China

Edward Narayan,

Western Sydney University, Australia

*Correspondence:

Shi Zhao

zhaoshiwuhan@126.com

Specialty section:

This article was submitted to

Experimental Endocrinology,

a section of the journal

Frontiers in Endocrinology

Received: 23 April 2019

Accepted: 18 July 2019

Published: 06 August 2019

Citation:

Wei W, Zhao S, Fu S, Yi L, Mao H, Tan Q, Xu P and Yang G (2019) The

Association of Hypoglycemia

Assessed by Continuous Glucose

Monitoring With Cardiovascular Outcomes and Mortality in Patients

With Type 2 Diabetes.

Front. Endocrinol. 10:536.

doi: 10.3389/fendo.2019.00536

\author{
Wei Wei ${ }^{1}$, Shi Zhao ${ }^{1 *}$, Sha-li Fu ${ }^{1}$, Lan $\mathrm{Yi}^{1}$, Hong Mao ${ }^{1}$, Qin $\operatorname{Tan}^{2}$, Pan $X u^{3}$ and \\ Guo-liang Yang ${ }^{4}$
}

${ }^{1}$ Department of Endocrinology, Tongji Medical College, The Central Hospital of Wuhan, Huazhong University of Science and Technology, Wuhan, China, ${ }^{2}$ Department of Cardiology, Tongji Medical College, The Central Hospital of Wuhan, Huazhong University of Science and Technology, Wuhan, China, ${ }^{3}$ Department of Neurology, Tongji Medical College, The Central Hospital of Wuhan, Huazhong University of Science and Technology, Wuhan, China, ${ }^{4}$ Department of Information, Tongji Medical College, The Central Hospital of Wuhan, Huazhong University of Science and Technology, Wuhan, China

Objective: Hypoglycemia has been shown to promote inflammation, a common pathogenic process, in many chronic health conditions including diabetes and cardiovascular disease. The aim of this study was to investigate the association of hypoglycemia, assessed by continuous glucose monitoring (CGM) with major adverse cardiovascular event (MACE) outcomes and all-cause mortality.

Methods: A retrospective cohort study was conducted with 1,520 patients with type 2 diabetes mellitus (T2DM). The severity of hypoglycemia event was assessed by CGM system.

Results: Three hundred and forty-seven participants experienced hypoglycemia events (323 with mild hypoglycemia and 24 with severe hypoglycemia). A fraction of $72.62 \%$ hypoglycemia was asymptomatic. During a median follow-up of 31 months, 380 participants reached the primary outcome of MACE (61 cardiovascular death, 50 non-fatal myocardial infarction [MI], 116 non-fatal stroke, 153 unstable angina requiring hospitalization), 80 participants died before the end of the study. In multivariate Cox regression models, hypoglycemia was associated with cardiovascular death ( $\mathrm{HR}$ 2.642[95Cl\% 1.398-4.994]), non-fatal stroke (HR 1.813 [95Cl\% 1.110-2.960]) and all-cause mortality (HR 1.960 [95 Cl\% 1.124- 3.418]) after the full adjustment. Hypoglycemia was not associated with non-fatal $\mathrm{Ml}$ and unstable angina. The $\mathrm{HR}$ of severe hypoglycemia was higher than mild hypoglycemia for cardiovascular death. Patients with symptomatic and asymptomatic hypoglycemia had similar MACE outcomes and all-cause mortality.

Conclusions: CGM is effective to detect asymptomatic and nocturnal hypoglycemia. Hypoglycemia is associated with an increased risk of non-fatal stroke, cardiovascular related death, and total mortality. The cardiovascular mortality is dose-dependent on the severity of hypoglycemia.

Keywords: T2DM, hypoglycemia, CGM system, MACE, all-cause mortality 


\section{INTRODUCTION}

Chronic low grade inflammation is a common feature underlying many chronic diseases and conditions such as insulin resistance and cardiovascular disease. Studies have suggested that hypoglycemia is able to promote inflammatory processes in patients with or without diabetes $(1,2)$. Hypoglycemia is a frequent adverse effect of anti-diabetic therapy in diabetic patients, and severe hypoglycemia has been identified as a potential risk factor for cardiovascular events in patients with type 2 diabetes mellitus (T2DM). Previous studies have reported an increased hazard ratio (HR) for adverse cardiovascular outcomes and total mortality in diabetic patients with hypoglycemia (3-6). However, these conclusions were drawn from the secondary analyses of randomized clinical trials $(7,8)$ or retrospective analyses of medical claims databases $(9,10)$, which a clear definition of hypoglycemia was lacking. Continuous glucose monitoring (CGM) system is an effective tool for the assessment of hypoglycemic status, particularly for the asymptomatic nocturnal hypoglycemia (11). Using CGM system, researchers found out that hypoglycemia is more common in T2DM patients than previously thought (12), and the frequency of hypoglycemia in those previous studies may be underestimated.

In the present study, using glucose data collected from CGMs, we investigated the relationships of hypoglycemia with total mortality and major adverse cardiovascular events (MACE), including non-fatal stroke, non-fatal myocardial infarction (MI), unstable angina leading to hospitalization, and cardiovascular death.

\section{MATERIALS AND METHODS}

\section{Subjects}

In this retrospective cohort study, we recruited 1,520 patients admitted to our hospital between January 2013 and December 2017.

The inclusion criteria were: (1) T2DM according to 2013 American Diabetes Association standards (13). Patients with other types of diabetes, such as type 1 diabetes, gestational diabetes, and secondary diabetes were excluded from the study. (2) Patients without the acute phase of illness, such as acute coronary syndrome, uncontrolled infection, etc. (3) CGM (iPro ${ }^{\circledR} 2$ CGM System Gold, Medtronic MiniMed, Inc., Northridge, CA) was used to monitor blood glucose within 3 days of admission without changing medications before the patients were discharged from hospital.

The CGM devices used in this study continuously measured the glucose level in the interstitial fluids within the range of 2.2$22.2 \mathrm{mmol} / \mathrm{L}$ (40-400 $\mathrm{mg} / \mathrm{dL}$ ). The glucose level was determined every $5 \mathrm{~min}, 288$ times maximum per day. The CGM system was calibrated with blood glucometer measurements (ACCUCHEK $^{\circledR}$ Aviva, Roche, Mannheim, Germany) four times daily according to the manufacturer's instruction. Participants were instructed to keep a diary about the occurrence of hypoglycemic symptoms. The CGM system used in our study measured interstitial glucose in a blinded manner, and data analysis was performed after disconnection of the device.

This clinical study was approved by the ethics committee boad of The Central Hospital of Wuhan. All participants had signed the informed consent form during the enrollment and before the start the study.

\section{Clinical Data Collection}

A clinical data warehouse was created as a collaborative program between Wuhan Central Hospital (Wuhan, China) and Shanghai Lejiu Healthcare Technology Co., Ltd. Data were extracted every $24 \mathrm{~h}$ by Lex Clinical Data Application 3.2 (Shanghai Lejiu Healthcare Technology Co., Ltd) from the Hospital Health Information System (HIS) to a designated clinical data warehouse including admission/transfer/discharge, laboratory orders/results, medication orders, discharge summary, administration events, flow sheet entries, procedures, medical reports, etc. All original unstructured data (i.e., pathology report, radiology report, admit/discharge summary etc.) were exclusively converted to a uniformed structured format. Core elements of the data warehouse were completely de-identified so that all queries and analytics could be carried out without exposing the confidential health data, allowing the investigators with sufficient privilege to re-identify data. Lex Clinical Data Application 3.2 was a self-service data access tool designed to query the clinical data warehouse and return tabular data for analysis and visualization.

\section{Definition of Hypoglycemia}

A hypoglycemia event was defined as an interstitial glucose level below $3.9 \mathrm{mmol} / \mathrm{L}(70 \mathrm{mg} / \mathrm{dl})$ for at least $15 \mathrm{~min}$ and recovery when the interstitial glucose concentration had been continuously above the threshold for $15 \mathrm{~min}$ or more (11). Percent time at interstitial glucose level below $3.9 \mathrm{mmol} / \mathrm{L}$ was evaluated. A severe hypoglycemia event was defined as cognitive impairment requiring external assistance for recovery. A mild hypoglycemia event was defined as a glucose level below 3.9 $\mathrm{mmol} / \mathrm{L}$ without cognitive impairment and external assistance for recovery. Nocturnal hypoglycemia was defined as an episode occurring between 00:00 a.m. and 06:00 a.m.

\section{Follow-Ups and Outcomes}

After discharging from the hospital, participants were invited to join the out-patient blood glucose management system, which was run by the professional medical staff. Routine self-blood glucose (at least four times per week) and HbAlc (every 3 month) monitoring were demanded in order to know the glucose control condition and clinical medication. The median duration of follow-ups was 31 months (inter-quartile range, $22-56)$. Eighteen patients (1.18\%) with new episodes of severe hypoglycemic events during follow-up were excluded from the study, and $41(2.70 \%)$ patients were lost before follow-ups due to various reasons.

The primary outcome was the first occurrence of an adjudicated MACE, including non-fatal MI, non-fatal stroke, cardiovascular death, and unstable angina leading to hospitalization. The secondary outcome was death of any 
cause. The diagnoses of MACE outcomes were ascertained according to the hospitalization records, discharge summary and certification of death, which were adjudicated by an independent committee. The members of the committee were from the cardiovascular and neurology departments of our hospital, who were unaware of the CGM results. Follow-up time was calculated from the date of hypoglycemia event to the onset date of the MACE event, death, or end of study (31 August 2018). Cause of death was classified as cardiovascular death and all other causes of death.

\section{Statistical Analysis}

The differences between groups were compared using $t$-test for continuous variables and Chi-square test for categorical data. Cox proportional models were used to evaluate the association between hypoglycemia and either MACE or all-cause mortality. We progressively adjusted the models for potential confounders. Model 1 was a crude model. Model 2 included age, sex, eGFR, HbA1c, BMI, and duration of diabetes. Model 3 included all variables in model 2 plus smoking status, alcohol history, past medical history (hepatic disease, renal disease, malignancy, coronary heart disease, and stroke), all diabetic medications (insulin, sulfonylureas, metformin, alpha-glucosidase inhibitors, pioglitazone, glinides, and DPP-4 inhibitors), hypertension medication, lipid-lowering medication, and antiplatelet agents.

TABLE 1 | Baseline characteristics of the study population $(n=1,520)$.

\begin{tabular}{lc}
\hline Outcome & $\begin{array}{c}\text { Number of } \\
\text { patients }\end{array}$ \\
\hline Severity of hypoglycemia & \\
No hypoglycemia & $1173(77.17 \%)$ \\
Mild hypoglycemia & $323(21.25 \%)$ \\
$\quad$ Severe hypoglycemia & $24(1.58 \%)$ \\
Numbers of hypoglycemia events & \\
1 & $94(27.09 \%)$ \\
2 & $87(25.07 \%)$ \\
3 & $66(19.02 \%)$ \\
4 & $40(11.53 \%)$ \\
$\geq 5$ & $60(17.29 \%)$ \\
Symptoms of hypoglycemia & \\
Asymptomatic hypoglycemia & $265(76.37 \%)$ \\
Symptomatic hypoglycemia & $82(23.63 \%)$ \\
Time of hypoglycemia & \\
Nocturnal hypoglycemia & $155(44.67 \%)$ \\
Diurnal hypoglycemia & $192(55.33 \%)$ \\
MACE outcomes & 380 \\
Non-fatal myocardial infraction & $50(13.16 \%)$ \\
Non-fatal stroke & $116(30.53 \%)$ \\
Cardiovascular death & $61(16.05 \%)$ \\
Unstable angina requiring hospitalization & $153(40.26 \%)$ \\
All-cause mortality & 80 \\
\hline Detes &
\end{tabular}

Dates are presented as numbers (percentages).

MACE, major adverse cardiovascular event.
TABLE 2 | Clinical characteristics of participants by the occurrence of hypoglycemia.

\begin{tabular}{|c|c|c|c|}
\hline & $\begin{array}{c}\text { No hypoglycemia } \\
n=1,173\end{array}$ & $\begin{array}{c}\text { Hypoglycemia } \\
n=347\end{array}$ & $P$-value \\
\hline Age, years & $58.59 \pm 11.26$ & $62.27 \pm 11.58$ & $<0.001$ \\
\hline Gender, Male ( $n$ \%) & $592(50.5)$ & $191(55)$ & 0.142 \\
\hline Diabetes duration, years & $6.46 \pm 6.00$ & $7.78 \pm 7.37$ & 0.002 \\
\hline $\begin{array}{l}\text { Mean glucose of CGM, } \\
\mathrm{mmol} / \mathrm{L}\end{array}$ & $8.97 \pm 2.17$ & $7.81 \pm 2.06$ & $<0.001$ \\
\hline SD of CGM, mmol/L & $2.64 \pm 1.30$ & $3.29 \pm 1.71$ & $<0.001$ \\
\hline $\mathrm{FPG}, \mathrm{mmol} / \mathrm{L}$ & $9.15 \pm 3.81$ & $8.36 \pm 3.86$ & 0.001 \\
\hline $\mathrm{HbA} 1 \mathrm{c}, \%$ & $8.19 \pm 2.10$ & $7.73 \pm 1.96$ & $<0.001$ \\
\hline $\mathrm{BMI}, \mathrm{kg} / \mathrm{m}^{2}$ & $24.50 \pm 2.88$ & $24.75 \pm 2.90$ & 0.173 \\
\hline eGFR & $95.18 \pm 20.29$ & $89.09 \pm 21.78$ & 0.001 \\
\hline $\mathrm{TG}, \mathrm{mmol} / \mathrm{L}$ & $1.83 \pm 1.80$ & $1.84 \pm 1.58$ & 0.924 \\
\hline $\mathrm{TC}, \mathrm{mmol} / \mathrm{L}$ & $4.58 \pm 1.06$ & $4.62 \pm 1.02$ & 0.616 \\
\hline $\mathrm{HDL}, \mathrm{mmol} / \mathrm{L}$ & $2.61 \pm 0.85$ & $2.60 \pm 0.84$ & 0.750 \\
\hline $\mathrm{LDL}, \mathrm{mmol} / \mathrm{L}$ & $1.13 \pm 0.33$ & $1.14 \pm 0.29$ & 0.844 \\
\hline $\begin{array}{l}\text { Systolic blood pressure, } \\
\mathrm{mmHg}\end{array}$ & $130.46 \pm 17.03$ & $132.00 \pm 19.84$ & 0.154 \\
\hline $\begin{array}{l}\text { Diastolic blood pressure, } \\
\mathrm{mmHg}\end{array}$ & $78.67 \pm 9.78$ & $77.70 \pm 11.09$ & 0.116 \\
\hline Smoking status & $340(29)$ & $102(29.4)$ & 0.893 \\
\hline Alcohol history & $151(12.9)$ & $59(17)$ & 0.052 \\
\hline Previous hypoglycemia & $73(6.2)$ & $57(16.4)$ & $<0.001$ \\
\hline History of hepatic disease & $160(13.6)$ & $48(13.8)$ & 0.624 \\
\hline History of renal disease & $32(2.7)$ & $23(6.6)$ & 0.002 \\
\hline History of malignancy & $29(2.5)$ & $12(3.5)$ & 0.089 \\
\hline $\begin{array}{l}\text { History of coronary heart } \\
\text { disease }\end{array}$ & $191(16.3)$ & $66(19.0)$ & 0.254 \\
\hline History of stroke & $79(6.7)$ & $26(7.5)$ & 0.630 \\
\hline \multicolumn{4}{|l|}{ Diabetic Complication } \\
\hline Diabetic nephropathy & $331(28.2)$ & $106(30.5)$ & 0.418 \\
\hline Diabetic retinopathy & $283(24.1)$ & $91(26.2)$ & 0.436 \\
\hline $\begin{array}{l}\text { Diabetic peripheral } \\
\text { neuropathy }\end{array}$ & $246(21.0)$ & $78(22.5)$ & 0.551 \\
\hline $\begin{array}{l}\text { Peripheral arterial } \\
\text { disease }\end{array}$ & $8(0.7)$ & $4(1.2)$ & 0.487 \\
\hline \multicolumn{4}{|l|}{ Diabetic Medications } \\
\hline Insulin & $444(37.9)$ & $179(51.6)$ & 0.000 \\
\hline Sulfonylureas & $167(14.2)$ & $59(17.0)$ & 0.229 \\
\hline Metformin & $407(34.7)$ & $55(15.9)$ & $<0.001$ \\
\hline $\begin{array}{l}\text { Alpha-glucosidase } \\
\text { inhibitors }\end{array}$ & 437 (37.3) & $124(35.7)$ & 0.613 \\
\hline Pioglitazone & $112(9.5)$ & $19(5.5)$ & 0.016 \\
\hline Glinides & $73(6.2)$ & $23(6.6)$ & 0.802 \\
\hline DPP-4 inhibitors & $87(7.4)$ & $12(3.5)$ & 0.009 \\
\hline Hypertension medication & $527(44.9)$ & $176(50.7)$ & 0.058 \\
\hline $\begin{array}{l}\text { Lipid-lowering } \\
\text { medication }\end{array}$ & 269 (22.9) & $78(22.5)$ & 0.884 \\
\hline Antiplatelet agents & $358(30.5)$ & $132(38.0)$ & 0.009 \\
\hline
\end{tabular}

Continuous variables are shown as mean $\pm S D$. Categorical data are presented as numbers (percentages).

CGM, continuous glucose monitoring; SD, standard deviation; FPG, fasting plasma glucose; BMI, body mass index; GFR, glomerular filtration rate; TG, triglyceride; TC, total cholesterol; LDL, low density lipoprotein; HDL, high density lipoprotein; DPP-4, dipeptidylpeptidse 4. 
For further analysis, we evaluated the risk of MACE outcomes and total mortality according to the severity of hypoglycemia and the appearance of hypoglycemic symptoms. The severity of hypoglycemia was classified into three categories: no hypoglycemia, mild hypoglycemia, and severe hypoglycemia. The group of no hypoglycemia served as the reference group, and adjusted for models described before. The survival curves of the three groups were estimate by Kaplan-Meier method, and the homogeneity between survival curves was tested by log-rank test.

All analyses were performed using State software (version 13.1, Stata Corp, College Station, TX). $P<0.05$ was considered as statistical significance.

\section{RESULT}

\section{Characteristics of Study Population}

Baseline characteristics of the study population were presented in Table 1. The total number of patients included in the study was 1,520, with 347 (22.83\%) patients experiencing hypoglycemic events. A total of 1,028 hypoglycemic events were recorded, corresponding to $250 \mathrm{~h}$ in hypoglycemia status. Of all the hypoglycemia, 24 patients were with severe hypoglycemia and 323 with mild hypoglycemia. The overall fraction of asymptomatic hypoglycemia was $72.62 \%$, and the fraction of nocturnal hypoglycemia was $44.67 \%$.

Compared to patients without hypoglycemia, those who experienced hypoglycemia were significantly older, had a longer duration of diabetes, and a lower eGFR. They were more likely to have experienced hypoglycemia events previously. They also took less anti-diabetic medications of metformin, pioglitazone, and DPP-4 inhibitors; meanwhile they were more often treated with insulin and anti-platelet agents (Table 2). Patients with severe hypoglycemia were even older, and more likely to be a smoker. They experienced hypoglycemia earlier than those with mild hypoglycemia (Supplementary Table 1).

Patients with hypoglycemia were further divided into two groups according to the appearance of hypoglycemic symptoms. Compared to patients with symptomatic hypoglycemia, patients experiencing asymptomatic hypoglycemia had lower mean glucose of CGM and smaller glycemic variability, were more likely to experience nocturnal hypoglycemia, and had longer periods of hypoglycemic events (Supplementary Table 2). The other baseline clinical characteristics of patients with symptomatic hypoglycemia were similar to those with asymptomatic hypoglycemia, except that patients experiencing asymptomatic hypoglycemia had a higher proportion of diabetic peripheral neuropathy (Supplementary Table 3).

\section{Association Between Hypoglycemia and MACE Outcomes}

During the follow-up, 380 diabetic patients had developed MACE (61 cardiovascular deaths, 153 unstable angina requiring hospitalization, 50 non-fatal MI, 116 non-fatal strokes). Eighty patients died before the end of our study. Of the 347 patients with hypoglycemia, the median time between hypoglycemia events and MACE outcomes was 21 (inter-quartile range, 11-38) months.

The crude incidence of MACE outcomes in people with hypoglycemia was higher than the incidence in people without hypoglycemia. These estimated results still remained significant (model 2: HR 1.592, 95\%CI, 1.233-2.056; model 3: HR 1.615, 95\% CI, 1.239-2.106) after further adjustments for potential confounding factors.

TABLE 3 | Association between hypoglycemia and MACE outcomes and all-cause mortality.

\begin{tabular}{|c|c|c|c|c|c|c|}
\hline & \multicolumn{2}{|c|}{ Events/ N } & \multirow[b]{2}{*}{$p$} & \multirow{2}{*}{$\begin{array}{c}\text { Model } 1 \\
\text { HR } \\
(95 \% \mathrm{Cl})\end{array}$} & \multirow{2}{*}{$\begin{array}{c}\text { Model } 2 \\
\text { HR } \\
(95 \% \mathrm{Cl})\end{array}$} & \multirow{2}{*}{$\begin{array}{c}\text { Model } 3 \\
\text { HR } \\
(95 \% \mathrm{Cl})\end{array}$} \\
\hline & With hypoglycemia & Without hypoglycemia & & & & \\
\hline MACE & $117 / 333$ & $263 / 1,128$ & $<0.001$ & $\begin{array}{c}1.501 \\
(1.207,1.866)\end{array}$ & $\begin{array}{c}1.592 \\
(1.233,2.056)\end{array}$ & $\begin{array}{c}1.615 \\
(1.239,2.106)\end{array}$ \\
\hline Cardiovascular death & 23/333 & $38 / 1,128$ & 0.006 & $\begin{array}{c}2.033 \\
(1.211,3.413)\end{array}$ & $\begin{array}{c}2.652 \\
(1.433,4.914)\end{array}$ & $\begin{array}{c}2.642 \\
(1.398,4.994)\end{array}$ \\
\hline $\begin{array}{l}\text { Unstable angina requiring } \\
\text { hospitalization }\end{array}$ & $41 / 333$ & $112 / 1,128$ & 0.300 & $\begin{array}{c}1.226 \\
(0.857,1.753)\end{array}$ & $\begin{array}{c}1.172 \\
(0.774,1.774)\end{array}$ & $\begin{array}{c}1.218 \\
(0.794,1.869)\end{array}$ \\
\hline Non-fatal Ml & $18 / 333$ & $32 / 1,128$ & 0.030 & $\begin{array}{c}1.901 \\
(1.067,3.389)\end{array}$ & $\begin{array}{c}1.634 \\
(0.828,3.226)\end{array}$ & $\begin{array}{c}1.549 \\
(0.768,3.124)\end{array}$ \\
\hline Non-fatal stroke & $35 / 333$ & $81 / 1,128$ & 0.060 & $\begin{array}{c}1.691 \\
(1.144,2.499)\end{array}$ & $\begin{array}{c}1.755 \\
(1.099,2.803)\end{array}$ & $\begin{array}{c}1.813 \\
(1.110,2.960)\end{array}$ \\
\hline All-cause mortality & $34 / 333$ & $46 / 1,128$ & $<0.001$ & $\begin{array}{c}2.501 \\
(1.605,3.898)\end{array}$ & $\begin{array}{c}2.259 \\
(1.323,3.858)\end{array}$ & $\begin{array}{c}1.960 \\
(1.124,3.418)\end{array}$ \\
\hline
\end{tabular}

Hypoglycemia was modeled as a time-dependent exposure.

Model 1 was a crude model.

Model 2 included age, sex, eGFR, HbA1C, BMI, and duration of diabetes.

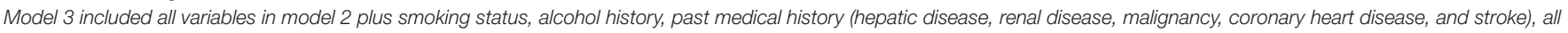

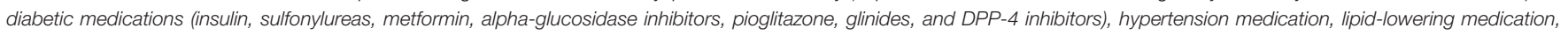
and antiplatelet agents.

MACE, major adverse cardiovascular event; MI, myocardial infarction. 
Furthermore, we examined the findings by subtypes of MACE outcomes. Compared to patients without hypoglycemia, those with hypoglycemia had a higher rate for non-fatal stroke, cardiovascular death and total mortality (Table 3). The associations were still persistent after additional adjustment in model 2 and model 3 . In the minimally adjusted models, hypoglycemia was associated with an increased risk of nonfatal MI, which was no longer observed after further adjustment (model 3: HR 1.549, 95\%CI 0.768-3.124). No association with hypoglycemia was found for unstable angina requiring hospitalization in any model.

Patients with severe hypoglycemia had a higher risk of cardiovascular death than those with mild hypoglycemia (Figure 1A). For subtypes of MACE outcomes, the values of HRs had a trend of rising in the severe hypoglycemia group compared with those in the hypoglycemia group, but the difference did not reach statistical significance (Figure 2). Patients with symptomatic and asymptomatic hypoglycemia had similar MACE outcomes and all-cause mortality (Figures 1B, 3).

\section{DISCUSSION}

The results of our study showed that hypoglycemia events detected by CGM were strongly associated with subsequent MACE outcomes and all-cause mortality. This association persisted after adjustment for a wide range of confounders. Furthermore, the risk of cardiovascular death and all-cause mortality was the highest after severe hypoglycemia events during CGM monitoring, especially in the first year, suggesting that health care providers should pay particular attention to the potential for morbidity and mortality after a severe hypoglycemic event. CGMs also detected a high proportion of asymptomatic hypoglycemic events, which appeared to have a similarly effect on MACE outcomes and all-cause mortality like the symptomatic hypoglycemia.

The Action to Control Cardiovascular Risk in Diabetes (ACCORD) study reported that intensive glycemic control was associated with increased risk of cardiovascular-related death $(3,4)$. Since the premature closure of the ACCORD study, the hypoglycemia-related cardiovascular adverse outcomes have led to considerable debate. The impacts of hypoglycemia on cardiovascular events in diabetic patients have been evaluated in several large prospective clinical trials, which have different conclusions $(5,6,14)$. Also, there were many observational studies, with inconsistent results of the association between hypoglycemia and MACE outcomes $(7,15)$. A subsequent metaanalysis including 10 studies suggested that severe hypoglycemia was associated with an almost two fold increased risk of cardiovascular events (17). Consistent with parts of those previous studies, the results of our analyses showed that hypoglycemia was associated with cardiovascular-related death and all-cause mortality. However, due to the insufficient cases of non-cardiovascular mortality $(n=19)$, we were unable to analyze the association between the cause-specific mortality and hypoglycemia like the Trail Comparing Cardiovascular Safety of Insulin Degludec vs. Insulin Glargine in Patients with Type 2
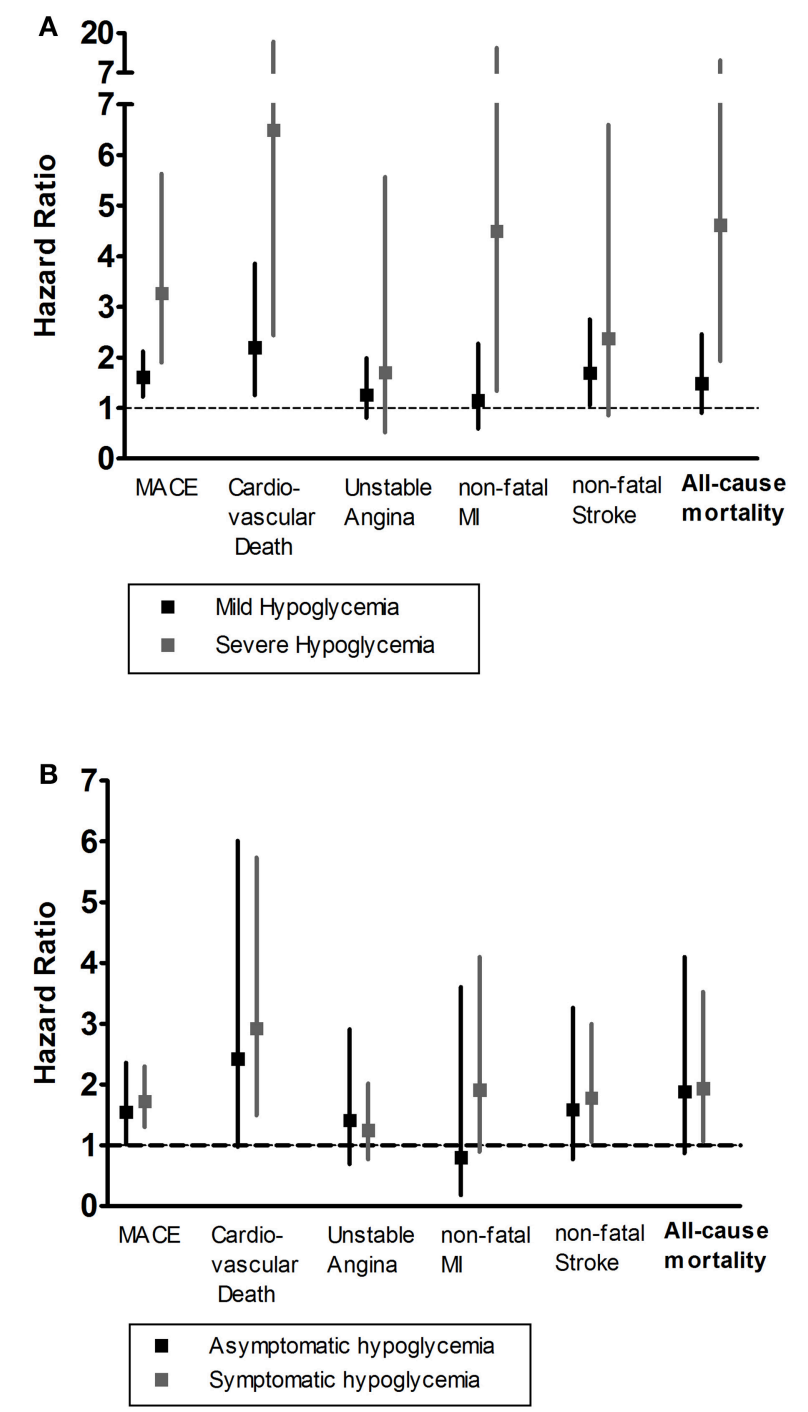

FIGURE 1 | (A) The HR and 95\% Cls of MACE outcomes and all-cause mortality according to the severity of hypoglycemia. (B) The $\mathrm{HR}$ and $95 \% \mathrm{Cls}$ of MACE outcomes and all-cause mortality according to the appearance of hypoglycemic symptoms. The severity of hypoglycemia was classified into three categories: no hypoglycemia, mild hypoglycemia, and severe hypoglycemia. The appearance of hypoglycemic symptoms was classified into three categories: no hypoglycemia, asymptomatic hypoglycemia, and symptomatic hypoglycemia. The group of no hypoglycemia served as the reference group, and adjusted for models including age, sex, duration of diabetes, HbA1c, BMI, eGFR, past medical history, diabetes medications, hypertension medication, lipid-lowering medication, and antiplatelet agents. HR, Hazard Ratios; MACE, major adverse cardiovascular event; MI, myocardial infarction.

Diabetes at High Risk of Cardiovascular Events (DEVOTE)study (16). In analysis of other subtypes of MACE outcomes, we can only see the association between hypoglycemia and an increased risk of developing non-fatal stroke. There was only a trend between hypoglycemia and non-fatal MI and unstable angina requiring hospitalization, which contrasted with the previous analyses $(10,14)$. 
A

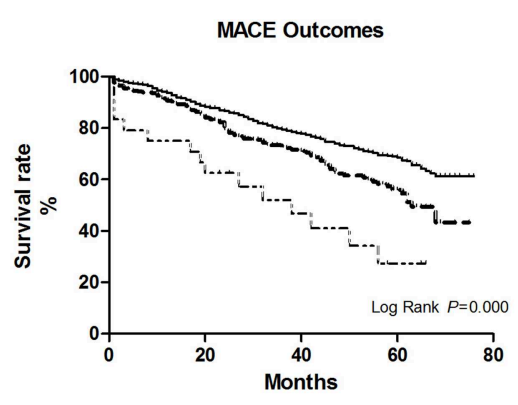

C

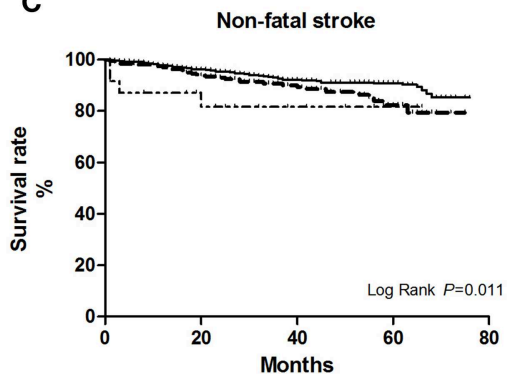

E

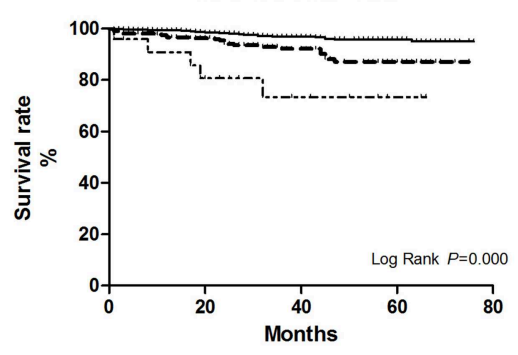

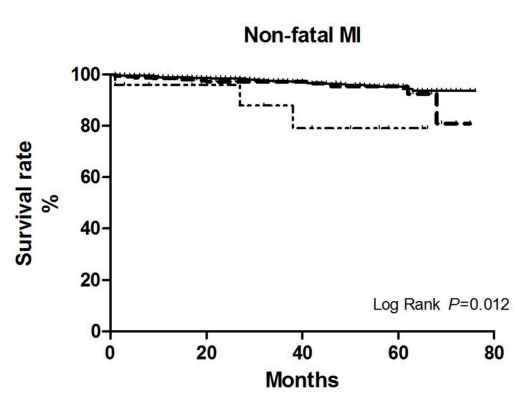

D
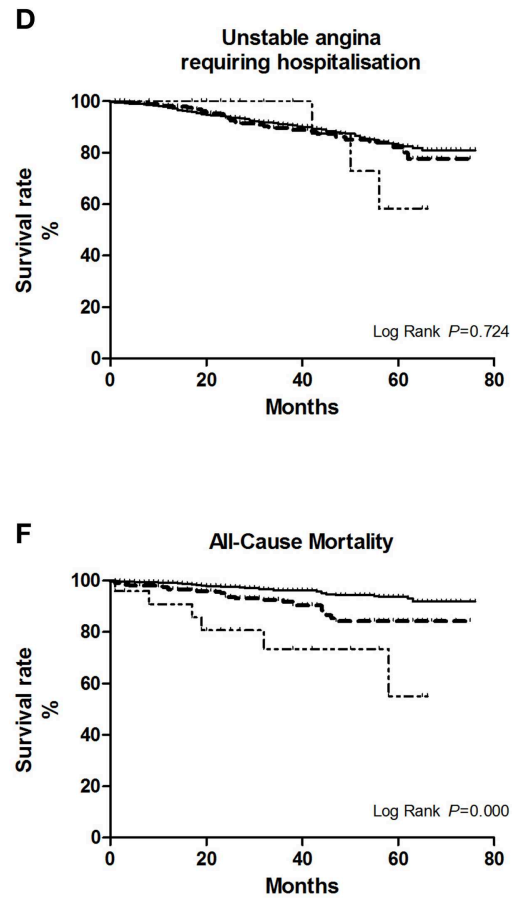

FIGURE 2 | The survival curves of the hypoglycemic severity were estimate by Kaplan-Meier method, and the homogeneity between survival curves was tested by log-rank test. (A) For overall MACE outcomes, the risk of MACE was the highest in the first year after severe hypoglycemia (25\%, 6/24). (B) For the subtype of non-fatal myocardial infarction (MI). (C) For the subtype of non-fatal stroke. (D) For the subtype of unstable angina leading to hospitalization. (E) For the subtype of cardiovascular death. (F) For all cause-mortality.

This can be explained by the different definitions of hypoglycemia, resulting in variable frequencies of hypoglycemia across studies. For most large epidemiological studies, hypoglycemia cases were collected by self-report $(5,6)$ or ICD-codes from medical electronic data $(9,10,14)$, which may underestimate the prevalence of hypoglycemia. In our study, a fraction of $76.37 \%$ hypoglycemic episodes were asymptomatic, which was also supported by earlier studies using CGM system $(18,19)$. Such a high proportion of asymptomatic hypoglycemia in diabetic patients with reduced awareness is worth our serious attention in clinical management. CGM is an effective way to detect hypoglycemia events, especially nocturnal and asymptomatic hypoglycemia, which could play an important role in reducing hypoglycemia events and is worth promoting in clinical applications.

We found some evidence of a dose-dependent relationship between the severity of hypoglycemia and cardiovascular death and all-cause mortality. Our assumption is that the cardiovascular outcomes of severe hypoglycemia may be worse than that of mild hypoglycemia. A sub-analysis of the ACCORD 


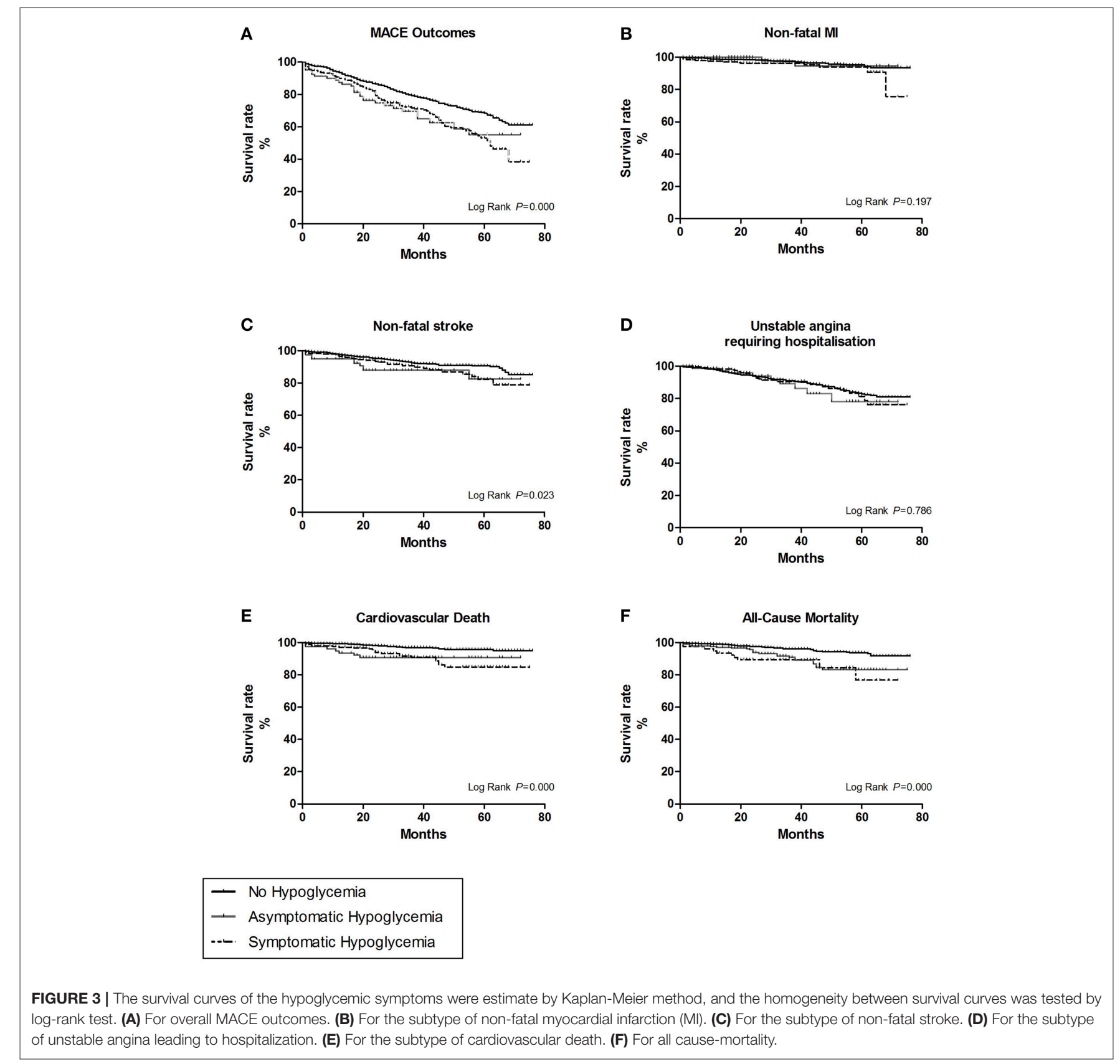

study found that the protective effect of recurrent mild hypoglycemia was more pronounced than severe hypoglycemia (20). It is suggested that exposure to mild hypoglycemia may offer better preparation against the adverse cardiovascular outcomes caused by severe hypoglycemia through prior blunting of sympathetic responses (21). In contrast to the findings of our study, several observational studies $(22,23)$ found out that mild hypoglycemia events have no association with mortality. Differences in methods of defining mild hypoglycemia may contribute to discrepancies. However, given the small number of participants with severe hypoglycemia $(n=24)$ in our study, these results may be limited by low statistical power.
The clinical management of T2DM emphasizes the importance of glycemic control to reduce the risk of chronic complications associated with diabetes (24). However, a toointensive glucose management therapy also puts patients at increased risk of hypoglycemia, which could be life-threatening. Given the concern that hypoglycemia might be a risk factor for cardiovascular disease, avoiding hypoglycemia remains a significant goal in optimizing glucose control. Individualizing glycemic targets should be considered for people with T2DM who are at high risk for hypoglycemia.

Currently, the standard of care in clinical practice is self-monitoring of capillary blood glucose (SMBG), 
which only provides a single point of time measurement and often fails to detect nocturnal and asymptomatic hypoglycemia. With the ability to measure glucose levels continuously and reflect glycemic variability, CGM technology is gaining increasing interest in clinical management. Numerous studies using CGM have demonstrated significant improvements in reducing hypoglycemia $(25,26)$. Future incorporation of CGMs in large clinical trials may provide precise information on the severity of hypoglycemia, as well as the glucose level at the occurrence of a hypoglycemic event.

Our study has two important strengths. First, to our knowledge, this is the first study of applying CGM to reveal the relationship between hypoglycemia and the increased risk of CVD. Previous epidemiologic investigations without an accurate definition of hypoglycemia may underestimate the prevalence of hypoglycemia events. Both the severity and time of hypoglycemia can be collected precisely through the CGM system to make a precise diagnosis of hypoglycemia. Second, we were able to adjust for numerous standardized and high-quality covariates, including the duration of diabetes, personal habits (smoking and drinking), BMI, and kidney function. In most large clinical trials, the ICD-code extracted from electronic medical records may be inaccurate, possibly leading to misclassification of exposure and confounding factors.

There were several limitations in our research. Firstly, the number of events for some outcomes may limit the precision of our estimations. Secondly, our study had a relatively short duration of follow-up with a median time of 31 months, which may limit the power to detect a significant association. Thirdly, the study only recruited participants of T2DM during hospitalization, which may not be generalizable beyond this population. Finally, the retrospective nature of our study precludes the possibility to explain the direct causal effect between hypoglycemia and MACE outcomes. Thus, welldesigned prospective cohort studies with the primary intention are needed to evaluate the association between hypoglycemia and cardiovascular outcomes.

In conclusion, through the analysis of glucose data collected by using CGMs, our results add to the accumulating evidence that hypoglycemia is associated with an increased risk of non-fatal stroke, cardiovascular death, and all-cause mortality. We also revealed a dose-dependent relationship between the severity of hypoglycemia and cardiovascular outcomes. Therefore, effective measurements should be taken to prevent severe hypoglycemia

\section{REFERENCES}

1. Gogitidze Joy N, Hedrington MS, Briscoe VJ, Tate DB, Ertl AC, Davis SN. Effects of acute hypoglycemia on inflammatory and pro-atherothrombotic biomarkers in individuals with type 1 diabetes and healthy individuals. Diabetes Care. (2010) 33:1529-35. doi: 10.2337/dc09-0354

2. Ratter JM, Rooijackers HM, Tack CJ, Hijmans AG, Netea MG, de Galan BE, et al. Proinflammatory effects of hypoglycemia in humans with or without diabetes. Diabetes. (2017) 66:1052-61. doi: 10.2337/db 16-1091 in patients with T2DM, especially those at high risk of cardiovascular problems.

\section{DATA AVAILABILITY}

The raw data supporting the conclusions of this manuscript will be made available by the authors, without undue reservation, to any qualified researcher.

\section{ETHICS STATEMENT}

This clinical study was approved by the ethics committee broad of The Central Hospital of Wuhan. All participants had signed the informed consent form during the enrollment and before the start the study.

\section{AUTHOR CONTRIBUTIONS}

WW and SZ conceived and designed the study. WW conducted the statistical analyses and wrote the manuscript. SZ provided guidance for the statistical analysis and made critical revisions to the manuscript for important intellectual content. HM provided guidance for the statistical analysis. GY collaborated with Shanghai Lejiu Healthcare Technology Co., Ltd. and took responsibility for the integrity of the data. QT and PX adjudicated the MACE outcomes and the cause of death. LY and SF took charge of the patients' follow-up.

\section{FUNDING}

This work was supported by the National Natural Science Foundation of China [No. 81370942] and the project of Regenerative Medicine Clinical Research Center of Hubei Province.

\section{ACKNOWLEDGMENTS}

The authors thank the participants of this study for their contributions.

\section{SUPPLEMENTARY MATERIAL}

The Supplementary Material for this article can be found online at: https://www.frontiersin.org/articles/10.3389/fendo. 2019.00536/full\#supplementary-material 
6. Duckworth WC, Abraira C, Moritz TE, Davis SN, Emanuele N, Goldman $\mathrm{S}$, et al. The duration of diabetes affects the response to intensive glucose control in type 2 subjects: the VA diabetes trial. J Diabetes Compl. (2011) 25:355-61. doi: 10.1016/j.jdiacomp.2011.10.003

7. ORIGIN Trial Investigators, Mellbin LG, Rydén L, Riddle MC, Probstfield J, Rosenstock J, et al. Does hypoglycaemia increase the risk of cardiovascular events? A report from the ORIGIN trial. Eur Heart J. (2013) 34:313744. doi: 10.1093/eurheartj/eht332

8. Heller SR, Bergenstal RM, White WB, Kupfer S, Bakris GL, Cushman WC, et al. Relationship of glycatedhaemoglobin and reported hypoglycaemia to cardiovascular outcomes in patients with type 2 diabetes and recent acute coronary syndrome events: the EXAMINE trial. Diabetes Obes Metab. (2017) 19: 664-71. doi: 10.1111/dom.12871

9. Hsu PF, Sung SH, Cheng HM, Yeh JS, Liu WL, Chan WL, et al. Association of clinical symptomatic hypoglycemia with cardiovascular events and total mortality in type 2 diabetes: a nationwide population-based study. Diabetes Care. (2013) 36: 894-900. doi: 10.2337/dc12-0916

10. Goto A, Goto M, Terauchi Y, Yamaguchi N, Noda M. Association between severe hypoglycemia and cardiovascular disease risk in Japanese patients with type 2 diabetes. J Am Heart Assoc. (2016) 5:e002875. doi: 10.1161/JAHA.115.002875

11. Danne T, Nimri R, Battelino T, Bergenstal RM, Close KL, DeVries JH, et al. International consensus on use of continuous glucose monitoring. Diabetes Care. (2017) 40:1631-40. doi: 10.2337/dc17-1600

12. Gehlaut RR, Dogbey GY, Schwartz FL, Marling CR, Shubrook JH. Hypoglycemia in type 2 diabetes-more common than you think: a continuous glucose monitoring study. J Diabetes Sci Technol. (2015) 9:9991005. doi: 10.1177/1932296815581052

13. American Diabetes Association. Standards of medical care in diabetes-2013. Diabetes Care. (2013) 36(Suppl. 1):S11-66. doi: 10.2337/dc13-S011

14. Lee AK, Warren B, Lee CJ, McEvoy JW, Matsushita K, Huang ES, et al. The association of severe hypoglycemia with incident cardiovascular events and mortality in adults with type 2 diabetes. Diabetes Care. (2018) 41:10411. doi: $10.2337 / \mathrm{dc} 17-1669$

15. Khunti K, Davies M, Majeed A, Thorsted BL, Wolden ML, Paul SK. Hypoglycemia and risk of cardiovascular disease and all-cause mortality in insulin-treated people with type 1 and type 2 diabetes: a cohort study. Diabetes Care. (2015) 38:316-22. doi: 10.2337/dc14-0920

16. Pieber TR, Marso SP, McGuire DK, Zinman B, Poulter NR, Emerson SS, et al. DEVOTE 3: temporal relationships between severe hypoglycemia, cardiovascular outcomes and mortality. Diabetologia. (2018) 61:58-65. doi: 10.1007/s00125-017-4422-0

17. Goto A, Arah OA, Goto M, Terauchi Y, Noda M. Severe hypoglycaemia and cardiovascular disease: systematic review and meta-analysis with bias analysis. BMJ. (2013) 347:f4533. doi: 10.1136/bmj.f4533

18. Henriksen MM, Andersen HU, Thorsteinsson B, Pedersen-Bjergaard U. Hypoglycemic exposure and risk of asymptomatic hypoglycemia in type 1 diabetes assessed by continuous glucose monitoring. J Clin Endocrinol Metab. (2018) 103: 2329-35. doi: 10.1210/jc.2018-00142

19. Uemura F, Okada Y, Torimoto K, Tanaka Y. Relation between hypoglycemia and glycemic variability in type 2 diabetes patients with insulin therapy: a study based on continuous glucose monitoring. Diabetes Technol Ther. (2018) 20: 140-6. doi: 10.1089/dia.2017.0306

20. Seaquist ER, Miller ME, Bonds DE, Feinglos M, Goff DC Jr, Peterson K, et al. The impact of frequent and unrecognized hypoglycemia on mortality in the ACCORD study. Diabetes Care. (2012) 35:409-14. doi: 10.2337/ dc11-0996

21. Reno CM, Daphna-Iken D, Chen YS, Vander Weele J, Jethi K, Fisher SJ. Severe hypoglycemia-induced lethal cardiac arrhythmias are mediated by sympathoadrenal activation. Diabetes. (2013) 62:3570-81. doi: 10.2337/db13-0216

22. McCoy RG, Van Houten HK, Ziegenfuss JY, Shah ND, Wermers RA, Smith SA. Increased mortality of patients with diabetes reporting hypoglycemia. Diabetes Care. (2012) 35:1897-901. doi: 10.2337/dc11-2054

23. Luk AO, Ho TS, Lau ES, Ko GT, Ozaki R, Tsang CC, et al. Association of self-reported recurrent mild hypoglycemia with incident cardiovascular disease and all-cause mortality in patients with type 2 diabetes: prospective analysis of the Joint Asia diabetes evaluation registry. Medicine. (2016) 95:e5183. doi: 10.1097/MD.0000000000005183

24. Holman RR, Paul SK, Bethel MA, Matthews DR, Neil HA. 10-year followup of intensive glucose control in type 2 diabetes. N Engl J Med. (2008) 359:1577-89. doi: 10.1056/NEJMoa0806470

25. Bolinder J, Antuna R, Geelhoed-Duijvestijn P, Kröger J, Weitgasser R. Novel glucose-sensing technology and hypoglycaemia in type 1 diabetes: a multicentre, non-masked, randomised controlled trial. Lancet. (2016) 388:2254-63. doi: 10.1016/S0140-6736(16) 31535-5

26. Haak T, Hanaire H, Ajjan R, Hermanns N, Riveline JP, Rayman G. Flash glucose-sensing technology as a replacement for blood glucose monitoring for the management of insulin-treated type 2diabetes: a multicenter, open-label randomized controlled trial. Diabetes Ther. (2017) 8:55-73. doi: 10.1007/s13300-016-0223-6

Conflict of Interest Statement: The authors declare that the research was conducted in the absence of any commercial or financial relationships that could be construed as a potential conflict of interest.

Copyright (C) 2019 Wei, Zhao, Fu, Yi, Mao, Tan, Xu and Yang. This is an open-access article distributed under the terms of the Creative Commons Attribution License (CC $B Y)$. The use, distribution or reproduction in other forums is permitted, provided the original author(s) and the copyright owner(s) are credited and that the original publication in this journal is cited, in accordance with accepted academic practice. No use, distribution or reproduction is permitted which does not comply with these terms. 